\title{
The Origin of the 100,000 Year Cycle in a Simple Ice Age Model
}

\author{
ROELOF K. SNIEDER ${ }^{1}$ \\ NOAA Geophysical Fluid Dynamics Laboratory, \\ Princeton University, Princeton, New Jersey
}

\begin{abstract}
A one-dimensional nonlinear ice age model developed by J. Imbrie and J. Z. Imbrie was used to investigate the 100,000 year cycle in climate records. It was already known that the model could mimic the 100,000 year cycle in the climate response, but it was not clear how this strong response was created. It is shown in this paper that the interference between the spectral components in the solar heating of 19,000 years and 23,000 years gives rise to an amplitude modulation, and how this modulation is converted by the nonlinearity to a 100,000 year cycle in the climate response.
\end{abstract}

\section{INTRODUCTION}

The spectral properties of climate records have received considerable interest in geophysical research. Geological data show fairly conclusive evidence that the climate variations in the past have a pronounced spectral component corresponding to a cycle of about $100,000 \mathrm{~K}$. (In this paper, time will always be measured in units of 1000 years, which will be denoted by $K=1000$ years.) These climate variations are thought to be paced by variations in the orbital parameters of the earth [see Hays et al., 1976; Imbrie and Imbrie, 1980].

It turns out, however, that the fluctuations in the earth's orbital parameters give rise to variations in the effective solar heating which have dominant periods only of 19,23 , and 41 $\mathrm{K}$, [see Berger, 1977]. (The 19 and $23 \mathrm{~K}$ cycle are caused mostly by the combined effects of eccentricity variations and precession; the $41 \mathrm{~K}$ cycle is caused by obliquity variations.) The $100 \mathrm{~K}$ cycle in climate records cannot be explained by variations in the earth's orbital parameters if the climate responds linearly to the solar heating. However, in a nonlinear climate system the nonlinearity could give rise to an energy transfer within the spectrum, which could create a strong 100 $\mathrm{K}$ cycle in the climate response.

Wigley [1976] showed this explicitly for a simple nonlinear equilibrium model which essentially squared the forcing. Birchfield [1977] showed the same behavior both for a "half wave rectifier" equilibrium model, as well as for Weertman's ice sheet model. In this paper the $100 \mathrm{~K}$ cycle in the model of Imbrie and Imbrie is discussed [Imbrie and Imbrie, 1980, heareafter referred to as II.]

The model of II is not an equilibrium model; a phase lag between the radiative forcing and the climate response is allowed. Their model is simple enough to understand what mechanism transfers energy to the $100 \mathrm{~K}$ cycle. II introduce the nonlinearity by assuming that ice sheets decay faster than they grow. (In II, several reasons are given to support this assumption.) If the solar heating is denoted by a function $x(t)$, and if the climate response is denoted by a function $y(t)$, then the ice sheet effect was parameterized by II as

\footnotetext{
${ }^{1}$ Now at Department of Theoretical Geophysics, University of Utrecht, The Netherlands.

Copyright 1985 by the American Geophysical Union.

Paper number 5D0095.

0148-0227/85/005D-0095\$05.00
}

$$
\begin{array}{ll}
\frac{d y}{d t}=\frac{1+b}{T_{m}}(x-y) & x \geq y \\
\frac{d y}{d t}=\frac{1-b}{T_{m}}(x-y) & x<y
\end{array}
$$

where $y(t)$ is taken as a negative measure of ice volume.

II found a strong $100 \mathrm{~K}$ cycle in the climate response $y(t)$, by using a realistic heating function $x(t)$ which contained most energy in cycles of 19,23 , and $41 \mathrm{~K}$. They found the best fit with observed climate variations of the last $150 \mathrm{~K}$ by choosing the following parameter setting: $T_{m}=17 \mathrm{~K}, b=0.6$. This means that the II system is highly nonlinear. It was, however, not clear why this nonlinearity gave rise to a very strong 100 $\mathrm{K}$ cycle in the response.

The goal of this paper is to clarify the source of the $100 \mathrm{~K}$ cycle. In order to do this, the model was integrated using several different heating functions $x(t)$. The spectra of the responses were used to trace down the origin of the $100 \mathrm{~K}$ cycle.

\section{Methods of ANalysis}

The heating functions that have been used in this study are superpositions of three oscillations:

$$
x(t)=A_{1} \cos \omega_{1} t+A_{2} \cos \omega_{2} t+A_{3} \cos \omega_{3} t
$$

The frequencies $\omega_{1}, \omega_{2}$, and $\omega_{3}$ correspond to periods of 19 , 23 , and $41 \mathrm{~K}$. Equation (1) was integrated for $700 \mathrm{~K}$; the last $655.5 \mathrm{~K}$ were used to determine the spectral density of the response. The time step and the record length were chosen in such a way that the spectral density was calculated for $\omega=2 \pi / 655.5 \mathrm{~K}$ and multiples of this frequency, so that the spectral density was evaluated for a period of $109 \mathrm{~K}$. The parameters $T_{m}$ and $b$ had the same values as in II, that is, $T_{m}=17 \mathrm{~K}$ and $b=0.6$, except in the last experiment, where the nonlinearity parameter $(b)$ was varied.

The mean of the response was subtracted in order to remove undesirable low-frequency effects. Next, the response was windowed [see Blackman and Tukey, 1958] and then Fourier transformed. The plots of the spectral density were normalized with respect to the maximum of the spectral components.

\section{Response to HaRmonic Forcing Functions}

The system (1) was first integrated with heating functions that contained only one frequency component, that is, only 


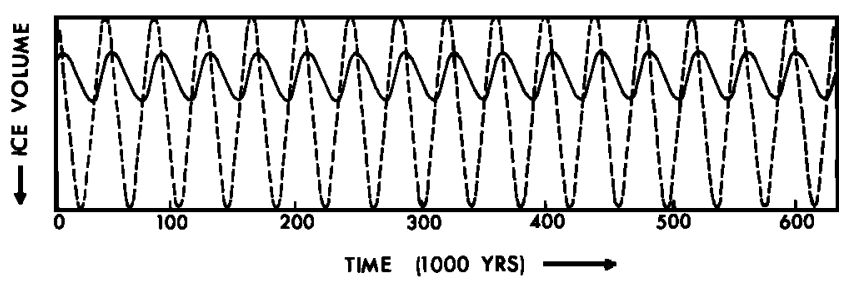

Fig. 1. Time series of the heating (dashed line) and the climate response (solid line) for a harmonic forcing with a period of $41 \mathrm{~K}$.

one of the $A_{\imath}$ in (2) was different from zero. This will be called a harmonic heating function. As an example, time series of the heating and the climate response are shown in Figure 1. In this case the heating occurred only at $41 \mathrm{~K}$. The spectrum of the climate response is shown in Figure 2.

The main feature of the spectra of the responses to harmonic heating functions is the strong peak at the frequency at which the system is forced. The spectral energy of the climate response is contained within a narrow spectral band (or equivalently, the climate response is almost sinusoidal) despite the fact that the system is highly nonliner. This is because the climate adjusts itself in such a way that the time-averaged climate is warmer than it would be in the case of a constant solar heating with the same mean. This has the effect that the ice sheet spends about the same amount of time "growing" as it is "decaying," despite the fact that the time constants for growth and decay are quite different (see (1)).

The fact that the response is not exactly sinusoidal can be seen in Figure 2, where the overtones of the $41 \mathrm{~K}$ cycle can be seen at 20.5 and $13.67 \mathrm{~K}$. These peaks would be absent if the response were exactly sinusoidal.

The important point of the experiments with a harmonic forcing is that the spectrum of the response contains essentially only the frequency component of the forcing. This indicates that the strong $100 \mathrm{~K}$ response in the II model is not caused by any sort of internal resonance of the system. As already noted by Le Treut and Ghil [1983], this is because the model of II consists of only one first-order differential equation.

The equilibrium solution of the II system is given by $y_{\text {eq }}=$ $x$, as can be seen from (1). This means that the transient solution has a much smaller amplitude than the equilibrium solution (Figure 1) [see Held, 1982].

\section{RESPONSE TO ForCING FunCtions Containing Two Frequencies}

If a heating function that contains two frequency components is used, a more interesting response is generated. The

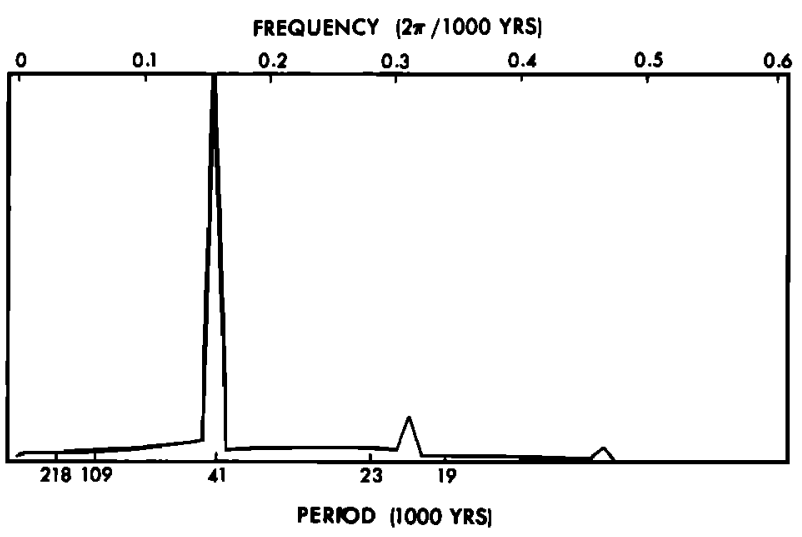

Fig. 2. Spectrum of the climate response in Figure 1. reason for this is that the linear superposition of two oscillations with equal amplitude gives rise to an amplitude modulation:

$$
\cos \left(\omega_{0}+\delta\right) t+\cos \left(\omega_{0}-\delta\right) t=2 \cos \delta t \cos \omega_{0} t
$$

Resulting in a modulation with a period given by

$$
T_{b}=\frac{2 \pi}{\delta}
$$

This time will be referred to as the "beat period." It has to be remembered that the function $\cos \delta t$ changes sign twice in every time interval of length $T_{b}$, so that the amplitude of the modulation has a period $T_{b} / 2$. This result also holds if the amplitudes of the two constitutive oscillations are not equal, although in that case, the modulation is weaker.

The beat period can be calculated by taking (3) and defining $T_{i}=2 \pi /\left(\omega_{0}-\delta\right)$ and $T_{j}=2 \pi /\left(\omega_{0}+\delta\right)$, so that

$$
T_{b}=2 T_{i} T_{j} /\left|T_{i}-T_{j}\right|
$$

A table of the beat period of three different frequency combinations that were studied is given below:

\begin{tabular}{ccccc} 
Case & $T_{1}$ & & $T_{2}$ & $T_{b}$ \\
\cline { 5 - 5 } 1 & 19 & & 23 & 218.5 \\
2 & 23 & & 41 & 104.8 \\
3 & 19 & 41 & 70.8
\end{tabular}

The time series of the heating and the climate response for case 1 is shown in Figure 3; the spectrum of the response is shown in Figure 4. The amplitude modulation is clearly visible in Figure 3. Note that the climate response depends only on the amplitude of the beat and is insensitive to the sign of the modulation. This is caused by the fact that the "growth time" of the ice sheets is so much larger than the "decay time." This has the effect that the climate becomes warmer whenever the amplitude of the beat increases, and this occurs with a period of $109 \mathrm{~K}$.

It is therefore not surprising that the most important feature of the spectrum is the strong spectral component at $109 \mathrm{~K}$. This component corresponds to an oscillation with a period of a half beat time. As already noted, the reason for this is that the climate response is only determined by the amplitude of the modulation, but not by the sign of the modulation. This modulation effect is so strong that the $109 \mathrm{~K}$ cycle contains even more energy than the 19 and $23 \mathrm{~K}$ cycles at which the system is forced.

Note that the II model behaves similar to the model of Wigley [1976] or the "half wave rectifier" model of Birchfield [1977]. In all these models the strong $100 \mathrm{~K}$ cycle is generated because a positive response is in some sense favored to a negative response. The only difference is that the models of Birchfield and Wigley show a strong response at the "sum frequency" (corresponding to a period of $10.4 \mathrm{~K}$ ), whereas this

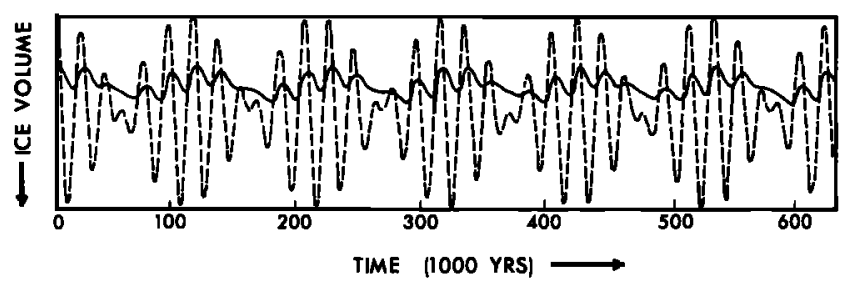

Fig. 3. Time series of the heating (dashed line) and the climate response (solid line) for a heating containing only oscillations with periods of 19 and $23 \mathrm{~K}$. 
spectral component is very weak in the II system. (This spectral component can just be seen at the end of the frequency scale in Figure 4.) This indicates that the treatment of parametric forcing of Le Treut and Ghil [1983] does not apply very well to the II system, since their theory predicts that the response at the sum frequency and the difference frequency will be of equal strength.

The spectra of the response to forcing functions containing frequencies at 19 and $41 \mathrm{~K}$ or 23 and $41 \mathrm{~K}$ are similar to Figure 4, except that the spectral components in the climate response due to the amplitude modulations are very weak. This is because the concept of amplitude modulation is only useful if

$$
\frac{1}{2} T_{b} \gg \max \left(T_{i}, T_{j}\right)
$$

This condition is certainly not satisfied for cases 2 and 3 (see the tabulation above).

\section{Variations of the Nonlinearity Parameter}

To see the role of the nonlinearity more clearly, several experiments were done in which the nonlinearity parameter (b) was varied, using the heating function of case 1 . This case was chosen because it gave rise to an extremely strong $109 \mathrm{~K}$ cycle. The heating and the climate response in the "very nonlinear case," that is, $b=0.9$, are shown in Figure 5. Note the nonlinear sawtooth-like response. The shape of the envelope of the climate response is not sinusoidal at all, resulting in a climate response that contains many overtones of the $109 \mathrm{~K}$ cycle. This has the effect that for $b=0.9$ there is less energy in the $109 \mathrm{~K}$ cycle than for $b=0.6$, despite the fact that the nonlinearity is stronger.

This effect can also be seen in Figure 6, in which the energy in the $109 \mathrm{~K}$ cycle is shown as a function of the nonlinearity parameter. For $b=0$ the $109 \mathrm{~K}$ cycle has zero energy, which is only to be expected for a linear system forced only at 19 and $23 \mathrm{~K}$. For $b=1$ the solution of $(1)$ is $y(t)=x_{\max }$, so that in that case the $109 \mathrm{~K}$ cycle does not contain any energy either. There clearly has to exist a maximum for intermediate $b$ values. It turns out that this maximum is attained for $b=0.72$.

It follows that II made a fortunate choice by assuming $b=0.6$, because this value is close to the value of $b$ that maximizes the energy in the $109 \mathrm{~K}$ cycle. (It is interesting to note that II determined their value of $b$ by fitting model results to real data of the last $150 \mathrm{~K}$ without considering any spectral component separately.)

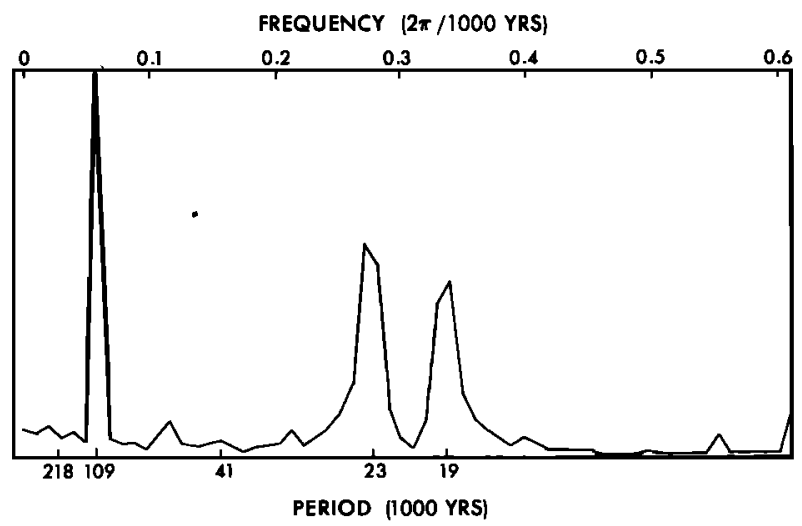

Fig. 4. Spectrum of the climate response in Figure 3.

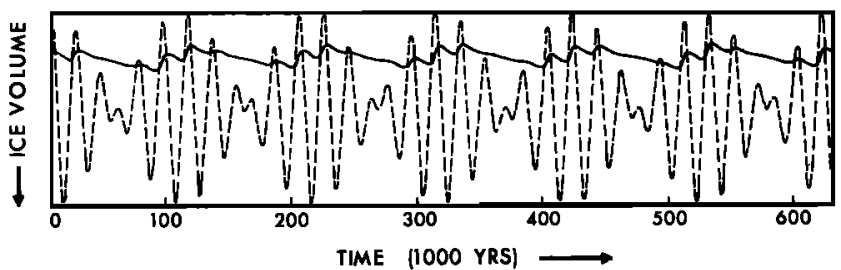

Fig. 5. Time series of the heating (dashed line) and the climate response (solid line) for the same heating as in Figure 3, but with $b=0.9$.

\section{Conclusion}

The nonlinear dynamical system of II has been integrated using heating functions that were superpositions of oscillations with periods of 19,23 , and $41 \mathrm{~K}$. The climate response to these heating functions contains essentially one frequency if the heating is harmonic. This confirms that the $109 \mathrm{~K}$ cycle in the response is not an internal resonance of the system [see Le Treut and Ghil, 1983].

If the system is forced with a heating function containing two frequency components, the climate response has a pronounced spectral component that is determined by the amplitude modulation of the heating. This effect is particularly strong if the system is forced at 19 and $23 \mathrm{~K}$.

The $109 \mathrm{~K}$ peak in the spectrum of the climate response of the II system appears therefore to be caused by an interference of the 19 and $23 \mathrm{~K}$ cycles, which gives rise to an amplitude modulation. The nonlinearity converts this amplitude modulation to a $109 \mathrm{~K}$ cycle. Just as in Wigley [1976] or in the half wave rectifier model of Birchfield [1977], this happens because a positive response is favored to a negative response. Note that the 19 and the $23 \mathrm{~K}$ cycles are present because the combined effects of eccentricity and precession lead to a splitting of the precession cycle into two cycles with periods of 19 and $23 \mathrm{~K}$ [see Hays et al., 1976]. The nonlinearity therefore extracts the variations in the eccentricity and transfers the energy from the 19 and $23 \mathrm{~K}$ cycles to the $109 \mathrm{~K}$ cycle. This means that it is ultimately the eccentricity cycle that generates the $109 \mathrm{~K}$ cycle in the climatic response.

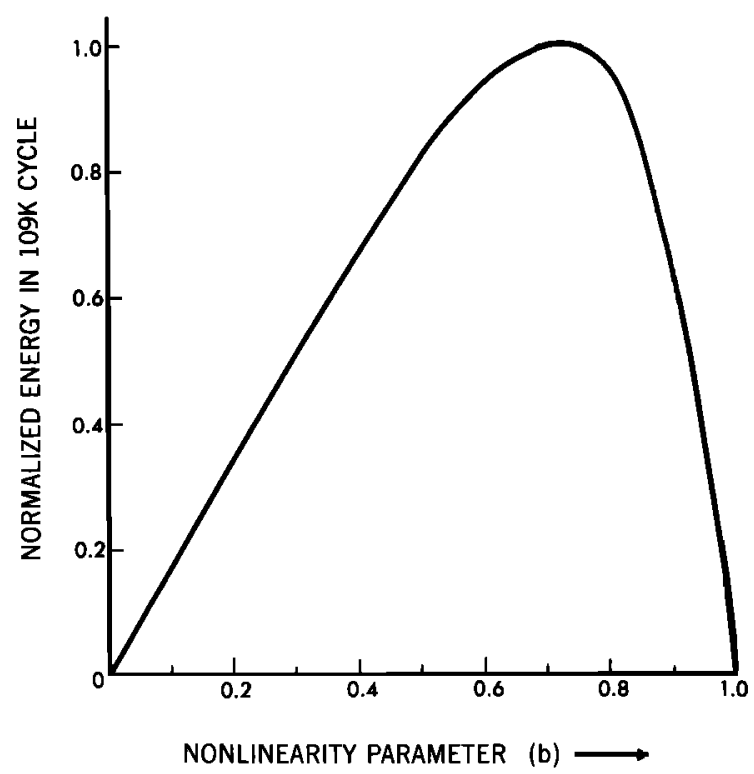

Fig. 6. Energy in the $109 \mathrm{~K}$ cycle in the climate response as a function of the nonlinearity parameter, for the heating shown in Figure 3. 
Acknowledgments. The author would like to thank A. Oort and S. Manabe for useful comments. The research was supported by grant ATM 8218761 A01 from the National Science Foundation.

\section{REFERENCES}

Berger, A. L., Support for the astronomical theory of climatic change, Nature, 269, 44-45, 1977.

Birchfield, G. E., A study of the stability of a model continental ice sheet subject to periodic variations in heat input, J. Geophys. Res., $82,4909-4913,1977$.

Blackman, R. B., and J. W. Tukey, The Measurement of Power Spectra, Dover, New York, 1958

Hays, J. D., J. Imbrie, and N. J. Shackleton, Variations in the earth's orbit: Pacemaker of the ice ages, Science, 194, 1121-1132, 1976.
Held, I. M., Climate models and the astronomical theory of the ice ages, Icarus, 50, 449-461, 1982.

Imbrie, J., and J. Z. Imbrie, Modeling the climatic response to orbital variations, Science, 207, 943-954, 1980.

Le Treut, $H$., and $M$. Ghil, Orbital forcing, climatic interactions, and glaciation cycles, J. Geophys. Res., 88, 5167-5190, 1983.

Wigley, T. M L., Spectral analysis and the astronomical theory of climatic change, Nature, 264, 629-631, 1976.

R. K. Snieder, Department of Theoretial Geophysics, University of Utrecht, Budapestlaan 4, P.O. Box 80.021, 3508 TA Utrecht, The Netherlands.

(Received April 30, 1984; revised January 29, 1985; accepted January 31,1985 .) 